\title{
Rare Ectopic Pregnancies - A Literature Review for the Period 2007-2019 on Locations Outside the Uterus and Fallopian Tubes
}

\section{Seltene extrauterine Schwangerschaften - eine Literaturübersicht 2007-2019 zu Lokalisationen außerhalb von Uterus und Tuben}

\section{(다)(i) (오 $\ominus$}

Authors

Sophie M. Eisner ${ }^{1}$, Andreas D. Ebert ${ }^{2}$, Matthias David ${ }^{1}$

Affiliations

1 Charité - Universitätsmedizin Berlin, Klinik für Gynäkologie, Campus Virchow Klinikum, Berlin, Germany

2 Praxis für Frauengesundheit, Gynäkologie und Geburtshilfe, Berlin, Germany

Key words ectopic pregnancy, rare locations, abdominal pregnancy

Schlüsselwörter

Extrauteringravidität, seltene Lokalisationen, Bauchhöhlenschwangerschaft

$\begin{array}{ll}\text { received } & 12.8 .2019 \\ \text { revised } & 7.4 .2020 \\ \text { accepted } & 19.5 .2020\end{array}$

Bibliography

DOI https://doi.org/10.1055/a-1181-8641

Geburtsh Frauenheilk 2020; 80: 686-701 @ Georg Thieme

Verlag KG Stuttgart · New York | ISSN 0016-5751

Correspondence

Prof. Dr. Matthias David

Charité - Universitätsmedizin Berlin, Klinik für Gynäkologie, Campus Virchow Klinikum

Augustenburger Platz 1, 13353 Berlin, Germany

matthias.david@charite.de

$\Theta$

Deutsche Version unter:

https://doi.org/10.1055/a-1181-8641

\section{ABSTRACT}

The majority of ectopic pregnancies (EP) are tubal pregnancies, but other implantation sites outside the uterus and tubes are also found. These rare EP locations present a particular diagnostic and therapeutic challenge. We present an overview of potential very rare locations of ectopic pregnancies, their symptoms, diagnosis and treatment, based on a systematic analysis of case reports. A literature review of the databases
PubMed, Livivo and Google Scholar for the period 2007 to 2019 was carried out. A total of 113 publications were included in our review. These studies describe EP implantations in the posterior cul-de-sac, on the uterine serosa and uterine ligaments, in the vicinity of almost all intraperitoneal organs, on the abdominal wall as well as in retroperitoneal sites. The most common presenting symptom was abdominal pain occurring in different locations. The diagnostic procedures included various imaging procedures and/or explorative surgery at different advanced stages of pregnancy. The most common and preferred option was laparotomy for surgical treatment. The placenta was successfully resected in the majority of cases. A rare EP location should be considered when making a differential diagnosis in patients of child-bearing age with abdominal pain.

\section{ZUSAMMENFASSUNG}

Das Gros extrauteriner Schwangerschaften (EUG) sind Tubargraviditäten, doch auch andere Implantationsstellen außerhalb des Uterus und der Adnexe kommen vor. Diese seltenen Lokalisationen einer EUG stellen eine besondere diagnostische und therapeutische Herausforderung dar. Anhand einer systematischen Analyse von Fallberichten wird eine Übersicht zu möglichen sehr seltenen Lokalisationen ektoper Schwangerschaften, deren Symptomatik, Diagnostik und Therapie gegeben. Dazu wurde eine Literaturrecherche in den Datenbanken PubMed, Livivo und Google Scholar für den Zeitraum 2007 bis 2019 durchgeführt. Insgesamt wurden 113 Publikationen eingeschlossen. Diese Studien beschrieben EUG-Implantationen im Douglas-Raum, an der Uterusserosa und dem uterinen Bandapparat, im Bereich nahezu aller intraperitonealer Organe, der Bauchwand sowie retroperitoneale Lokalisationen. Das häufigste Symptom war der variabel lokalisierte abdominale Schmerz. Die diagnostischen Maßnahmen umfassten unterschiedliche Bildgebungsverfahren und/oder explorative Operationen in verschieden weit fortgeschrittenem Schwangerschaftsalter. Als häufigste Therapieoption wurde die operative Befundsanierung per laparotomiam gewählt. Die Plazenta konnte in der Mehrheit der Fälle erfolgreich reseziert werden. Eine EUG seltener Lokalisation sollte 
als mögliche Differenzialdiagnose bei Patientinnen im reproduktionsfähigen Alter mit abdominellen Beschwerden bedacht werden.

\section{Introduction}

The frequency of ectopic pregnancies (EP) is estimated as 11 ectopic per 1000 (eutopic) pregnancies [1]. Despite improvements in diagnosis and treatment options, EP is still associated with a high risk of mortality and accounts for around $6-13 \%$ of all pregnancy-related deaths in the first trimester of pregnancy [2]. Tubal pregnancies are the most common form of EP with an incidence of $95 \%$, followed by implantation sites in the cervix, the ovary and the abdominal cavity [3]. Non-tubal EP are reported to have a 7-8 times higher risk of maternal mortality compared to tubal pregnancies $[4,5]$.

Known risk factors for non-tubal ectopic pregnancies include a previous history of EP, minor pelvic infections, pregnancy by assisted reproductive technology, and smoking [3]. Typical leading clinical symptoms of a ruptured EP are progressive pain in the lower abdomen, often accompanied by pronounced peritonism, shoulder area pain if hemoperitoneum is present, and acyclic vaginal bleeding, as well as dizziness, faintness and nausea [6]. As abdominal bleeding may be quite heavy, ectopic pregnancy is a real gynecological emergency because of the rapid onset of hemorrhagic shock. Vaginal ultrasound scan is usually carried out in patients with a positive pregnancy test to obtain the correct diagnosis as quickly as possible. In cases with EP, imaging will show a thickened endometrium but no intrauterine gestational sac or only a pseudo-gestational sac with no yolk sac [1]. In some cases, the implantation site in the adnexal area and fluid in the pouch of Douglas can be identified on imaging. The implantation site and the extent of symptoms determine the type of treatment and urgency of treatment. Particularly in cases with advanced abdominal EP, the question is often whether the ectopic placenta can be resected with limited bleeding or whether it can be left in situ. There are currently no guidelines by the AWMF (the Association of the Scientific Medical Societies in Germany) or the DGGG (the German Society for Gynecology and Obstetrics) on the appropriate approach to treat EP. Guidelines are planned and their completion has been announced for 2020 [7].

To obtain a rapid and correct diagnosis of an ectopic pregnancy in a rare location, it is important to be aware of the potential sites of implantation. Locations outside the uterus, tubes and ovaries are of particular interest, as they present a significant diagnostic challenge in emergency gynecological medicine because of the number of different potential implantation sites [8]. In 2007, Molinaro and Barnhart published a study "Ectopic pregnancies in unusual locations" [6]; in 2014 the Cochrane Foundation initiated a protocol for a not yet published systematic review [8]; and in 2016, Parker and Srinivas summarized the approaches for the management of non-tubal ectopic pregnancies [3]. These publications were almost exclusively based on case reports. We were unable to find a review which collected and summarized the known cases of non-tubal and non-ovarian EP. Case reports on rare locations were therefore collected for this review. As a review of the literature already existed [6], we selected the year 2007 as our chronological starting point. The aim of our literature search was to provide a comprehensive summary of the publications published since 2007 on rare locations of extrauterine pregnancies, their presenting symptoms, diagnosis and treatment.

\section{Methods}

\section{Inclusion and exclusion criteria}

A systematic search was carried out in the three online databases PubMed, Livivo and Google Scholar. English-language and German-language case reports from January 2007 onward which described an ectopic pregnancy in an unusual location were included in our review. Reports about uterine implantation sites (cervical and intramural pregnancies as well as scar pregnancies) were excluded as were ampullary, isthmic and interstitial tubal pregnancies and ovarian pregnancies. Pregnancies which occurred after hysterectomy and heterotopic pregnancies were not included.

\section{Search strategy}

The search was carried out in June 2019 for the period 2007-2019 using the MeSH terms "ectopic pregnancy" and "case report" and a filter for German-language and English-language publications. The subcategories "tubal pregnancy," "cornual pregnancy," "angular pregnancy," "ovarian pregnancy" and "scar pregnancy" were excluded from the search results using the operator "NOT."

This study complies with the statute of the Charite on ensuring good scientific practice.

\section{Results}

After carrying out a systematic literature search, a total of 115 suitable case reports from 113 publications were included in the study and their full text was analyzed ( $\bullet$ Fig. 1). Based on anatomical sites and pathophysiology, cases were divided into intraperitoneal and extraperitoneal EP locations. Intraperitoneal implantation sites included all locations on the uterine serosa, the uterine ligaments, the liver, spleen, and bowel including the mesentery and the peritoneum of the pelvic wall, the diaphragm and the inguinal canal. Reported extraperitoneal sites of EP implantation included sites on the large arteries, in the area of the pancreas and the kidneys, in rectovaginal and paravesical spaces and in the obturator foramen.

As regards the pathogenesis of intraperitoneal EP, it is important to distinguish between primary and secondary abdominal pregnancy. With primary abdominal pregnancy, it is assumed that fertilization and implantation of the ovum occurs in the peritoneal space as a result of the anatomical conditions at the open distal 


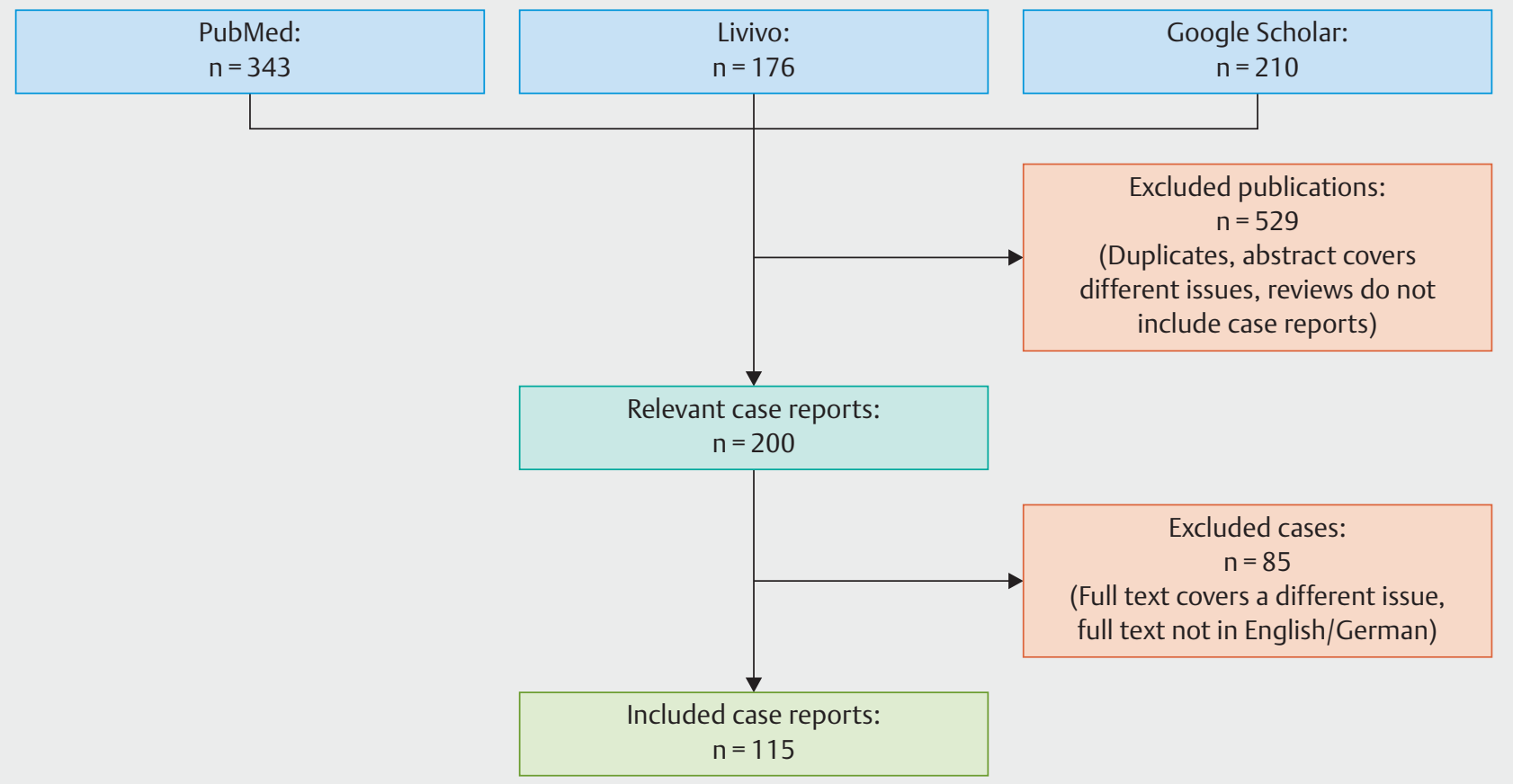

- Fig. 1 Flow chart showing the process and results of the literature search in the three databases.

portion of the uterine tube. In the case of secondary abdominal pregnancies, it is assumed that a secondary implantation in the peritoneal cavity occurs after rupture of a primary tubal pregnancy [3]. The pathogenesis of retroperitoneal EP, however, has not been conclusively established. Peritoneal fistulas following surgery [9] and lymphatic pathways [10] have been postulated as possible routes.

Presenting symptoms, diagnosis and treatment are summarized below, with cases grouped according to the location of the implantation site.

\section{A) Implantation in the uterine serosa and the pouch of Douglas ( Table 1 )}

Presenting symptoms

- Abdominal pain (14/32 cases), particularly in the lower abdomen; abdominal pain and respiratory problems (1/32); abdominal pain and vomiting (2/32); abdominal pain and back pain $(1 / 32)$

- Asymptomatic (10/32)

- Vaginal bleeding (2/32)

- Respiratory problems (1/32)

- Not specified (1/32)

\section{Diagnosis and diagnostic procedures}

- Earliest diagnosis made in the 5th week of gestation (GW) post last menstrual period, latest diagnosis in the 41st GW

- Diagnosed by ultrasound scan (11/32), exploratory laparoscopy (6/32), exploratory laparotomy (8/32), MRI (6/32) or at autopsy $(1 / 21)$

\section{Intervention}

- Surgical treatment with laparotomy (25/32), with additional administration of MTX in one case

- Surgical treatment with laparoscopy (6/32) with additional administration of MTX in 2 cases

- Not specified (2/32)

Placental management

- Resection of the placenta (23/32), with additional organ removal in 7 cases

- Placenta left in situ (7/32), with re-laparotomy with resection additionally performed in 2 cases

- Not specified (1/32) (autopsy)

It is worth noting that in many cases the pregnancy was already far advanced at diagnosis and many of the patients were asymptomatic. In 14/32 cases, a live infant was delivered by laparotomy. The most common site of implantation was the posterior uterine wall. 
- Table 1 Implantation in the uterine serosa and the pouch of Douglas.

\begin{tabular}{|c|c|c|c|c|c|c|c|c|c|c|}
\hline No. & Source & Age & GP score & GW & $\beta$-HCG & Symptoms & $\begin{array}{l}\text { Diagnostic } \\
\text { procedure }\end{array}$ & Intervention & $\begin{array}{l}\text { Placental } \\
\text { management }\end{array}$ & $\begin{array}{l}\text { Previous } \\
\text { medical } \\
\text { history }\end{array}$ \\
\hline 1 & $\begin{array}{l}\text { Abdelrahman, } \\
\text { 2017, South } \\
\text { Sudan [15] }\end{array}$ & 25 & G2P0 & 35 & - & $\mathrm{AbP}$ & exLtm & Ltm, live birth & Partial resection & - \\
\hline 2 & $\begin{array}{l}\text { Bhoil, 2016, } \\
\text { India [16] }\end{array}$ & 29 & G2P2 & 34 & - & $\mathrm{AbP}$ & MRI & Ltm, live birth & Resection & - \\
\hline 3 & $\begin{array}{l}\text { Bohiltea, 2015, } \\
\text { Romania [17] }\end{array}$ & 23 & GOPO & 23 & - & $\mathrm{AbP}$ & exLtm & Ltm & $\begin{array}{l}\text { Resection, uterine } \\
\text { wedge resection }\end{array}$ & IVF \\
\hline 4 & $\begin{array}{l}\text { Cho, 2015, } \\
\text { Taiwan [18] }\end{array}$ & 31 & GOPO & - & 40100 & VB & Sono & Lap, MTX & Left in situ & - \\
\hline 5 & $\begin{array}{l}\text { Dabiri, 2014, } \\
\text { USA [19] }\end{array}$ & 27 & G2P01 & 33 & - & $\mathrm{AbP}$ & exLtm & Ltm, live birth & $\begin{array}{l}\text { Resection, } \\
\text { hysterectomy }\end{array}$ & - \\
\hline 6 & $\begin{array}{l}\text { Dassah, 2009, } \\
\text { Ghana [20] }\end{array}$ & 21 & GOPO & 22 & - & LAbP & Sono & $\mathrm{Ltm}$ & Resection & - \\
\hline 7 & $\begin{array}{l}\text { Gayer 2012, } \\
\text { USA [21] }\end{array}$ & 30 & G2P1 & 19 & - & $x$ & MRI & Ltm & - & - \\
\hline 8 & $\begin{array}{l}\text { Gidiri, 2015, } \\
\text { Zimbabwe [22] }\end{array}$ & 40 & G4P3 & 21 & - & LAbP, BP & Sono & $\mathrm{Ltm}$ & Resection & - \\
\hline 9 & $\begin{array}{l}\text { Gidiri, 2015, } \\
\text { Zimbabwe [22] }\end{array}$ & 37 & $\mathrm{G} 2 \mathrm{P} 0+1$ & 35 & - & $\mathrm{AbP}$ & Sono & Ltm, live birth & Left in situ & $\begin{array}{l}\text { Myomec- } \\
\text { tomy }\end{array}$ \\
\hline 10 & $\begin{array}{l}\text { Hailu, 2017, } \\
\text { Ethiopia [4] }\end{array}$ & 26 & G4P2 & 37 & - & AbP, Vom. & exLtm & Ltm, live birth & Resection & - \\
\hline 11 & $\begin{array}{l}\text { Hishikawa, 2016, } \\
\text { Japan [23] }\end{array}$ & 32 & G3P1 & - & 120,60 & $\mathrm{AbP}$ & exLap & Lap & Resection & - \\
\hline 12 & $\begin{array}{l}\text { Isah, 2008, } \\
\text { Nigeria [24] }\end{array}$ & 30 & GOPO & 39 & - & VB & Sono & Ltm, live birth & Resection & - \\
\hline 13 & $\begin{array}{l}\text { Kim, 2013, } \\
\text { Korea [25] }\end{array}$ & 28 & G1P0 & 18 & - & $x$ & Sono & $\begin{array}{l}\text { Ltm (34th } \\
\mathrm{GW} \text { ) live birth }\end{array}$ & Resection & $\begin{array}{l}\text { Previous } \\
\text { EP }\end{array}$ \\
\hline 14 & $\begin{array}{l}\text { Marcelin, 2018, } \\
\text { France [26] }\end{array}$ & 25 & G2P0 & 27 & - & - & MRI & $\mathrm{Ltm}$ & $\begin{array}{l}\text { Left in situ, } \\
\text { embolization, } \\
\text { Lap because of } \\
\text { abscess formation }\end{array}$ & - \\
\hline 15 & $\begin{array}{l}\text { Miyauchi, 2015, } \\
\text { Japan [5] }\end{array}$ & 36 & G1P1 & 5 & 2050 & LAbP & exLap & Ltm & Resection & - \\
\hline 16 & $\begin{array}{l}\text { Mengistu, 2015, } \\
\text { Ethiopia [27] }\end{array}$ & 32 & G3P2 & 36 & - & Resp. & MRI & Ltm, live birth & $\begin{array}{l}\text { Resection, } \\
\text { hysterectomy }\end{array}$ & - \\
\hline 17 & $\begin{array}{l}\text { Muehlparzer, } \\
\text { 2011, Austria } \\
\text { [28] }\end{array}$ & 26 & - & 34 & - & LAbP & exLtm & $\begin{array}{l}\text { Ltm with live } \\
\text { birth }\end{array}$ & $\begin{array}{l}\text { Resection, } \\
\text { hysterectomy, } \\
\text { salpingectomy }\end{array}$ & - \\
\hline 18 & $\begin{array}{l}\text { Nassali, 2016, } \\
\text { Botswana [29] }\end{array}$ & 26 & GOPO & 41 & - & $\mathrm{AbP}$ & exLtm & $\begin{array}{l}\text { Ltm with live } \\
\text { birth }\end{array}$ & Resection & - \\
\hline 19 & $\begin{array}{l}\text { Nkusu, 2008, } \\
\text { Cameroon [30] }\end{array}$ & 30 & G5P5 & at term & - & $\mathrm{AbP}$ & Sono & $\mathrm{Ltm}$ & Resection & - \\
\hline 20 & $\begin{array}{l}\text { Panagiotakis, } \\
\text { 2009, USA [31] }\end{array}$ & 24 & GOPO & 7 & - & $\mathrm{AbP}$ & exLap & Lap, MTX & Left in situ & - \\
\hline 21 & $\begin{array}{l}\text { Parekh, 2008, } \\
\text { India [32] }\end{array}$ & 31 & - & 15 & - & LAbP & Sono & Ltm & $\begin{array}{l}\text { Resection, } \\
\text { hemostasis }\end{array}$ & - \\
\hline 22 & $\begin{array}{l}\text { Patel, 2016, } \\
\text { USA [33] }\end{array}$ & 26 & GOPO & 16 & - & LAbP & MRI & Ltm, MTX & $\begin{array}{l}\text { Left in situ, re-Ltm } \\
\text { with resection }\end{array}$ & - \\
\hline 23 & $\begin{array}{l}\text { Pieh-Holder, } \\
\text { 2012, USA [34] }\end{array}$ & 39 & G1P0 & 25 & - & $x$ & MRI & Ltm, live birth & $\begin{array}{l}\text { Left in situ, } \\
\text { embolization }\end{array}$ & $\begin{array}{l}\text { Myomec- } \\
\text { tomy }\end{array}$ \\
\hline
\end{tabular}


- Table 1 Implantation in the uterine serosa and the pouch of Douglas. (Continued)

\begin{tabular}{|c|c|c|c|c|c|c|c|c|c|c|}
\hline No. & Source & Age & GP score & GW & $\beta$-HCG & Symptoms & $\begin{array}{l}\text { Diagnostic } \\
\text { procedure }\end{array}$ & Intervention & $\begin{array}{l}\text { Placental } \\
\text { management }\end{array}$ & $\begin{array}{l}\text { Previous } \\
\text { medical } \\
\text { history }\end{array}$ \\
\hline 24 & $\begin{array}{l}\text { Pradhan, 2013, } \\
\text { India [35] }\end{array}$ & 35 & GOPO & 26 & - & AbP, Resp. & Autopsy & - & - & - \\
\hline 25 & $\begin{array}{l}\text { Promsonthi, } \\
\text { 2007, Thailand } \\
{[36]}\end{array}$ & 41 & G2P2 & 40 & - & $\mathrm{x}$ & exLtm & Ltm, live birth & $\begin{array}{l}\text { Left in situ, abscess } \\
\text { formation, Ltm, } \\
\text { hysterectomy, } \\
\text { salpingo-oopho- } \\
\text { rectomy }\end{array}$ & - \\
\hline 26 & $\begin{array}{l}\text { Rohilla, 2018, } \\
\text { India [37] }\end{array}$ & 27 & Multipara & 40 & - & $x$ & Sono & Ltm, live birth & $\begin{array}{l}\text { Resection, salpin- } \\
\text { go-oophorectomy }\end{array}$ & - \\
\hline 27 & $\begin{array}{l}\text { Sib, 2018, } \\
\text { Burkina Faso [38] }\end{array}$ & 22 & G4P2 & at term & - & $x$ & Sono & Ltm & $\begin{array}{l}\text { Resection, } \\
\text { salpingectomy }\end{array}$ & - \\
\hline 28 & $\begin{array}{l}\text { Shih, 2007, } \\
\text { Taiwan [39] }\end{array}$ & 33 & GOPO & $\begin{array}{l}20 \text { days } \\
\text { post ET }\end{array}$ & 901 & $\mathrm{x}$ & Sono & Lap & Resection by Ltm & IVF-ET \\
\hline 29 & $\begin{array}{l}\text { Tucker, 2017, } \\
\text { USA [40] }\end{array}$ & 28 & G2P1 & 22 & - & $x$ & exLap & Ltm & Resection & - \\
\hline 30 & $\begin{array}{l}\text { Yanaihara, 2017, } \\
\text { Japan [41] }\end{array}$ & 37 & P0 & 6 & - & $x$ & exLap & Lap & Resection & ICSI \\
\hline 31 & $\begin{array}{l}\text { Yoder, 2016, } \\
\text { USA [42] }\end{array}$ & 30 & G2P1 & $\begin{array}{l}33 \text { days } \\
\text { post ET }\end{array}$ & $\begin{array}{l}12400 \\
\mathrm{pg} / \mathrm{mL}\end{array}$ & $x$ & exLap & Lap & Resection & IVF-ET \\
\hline 32 & $\begin{array}{l}\text { Zhang, 2008, } \\
\text { China [43] }\end{array}$ & 30 & G3P1 & 38 & - & AbP, Vom. & exLtm & Ltm, live birth & $\begin{array}{l}\text { Resection, } \\
\text { hysterectomy }\end{array}$ & - \\
\hline \multicolumn{11}{|c|}{ 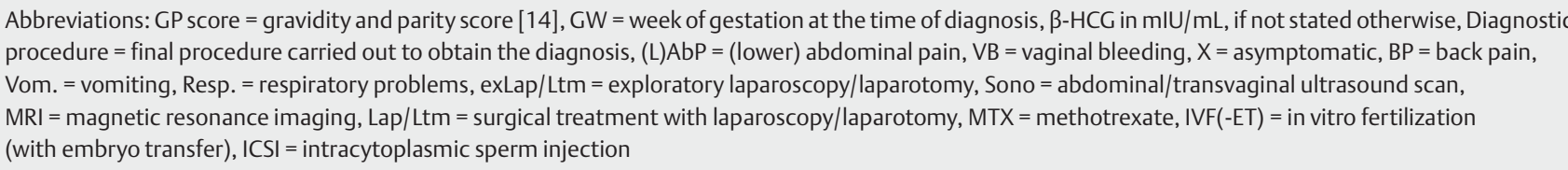 } \\
\hline
\end{tabular}

\section{B) Implantation on the broad ligament of uterus (- Table 2)}

Presenting symptoms

- Abdominal pain (6/18), particularly in the lower abdomen

- Abdominal pain and vaginal bleeding (4/18)

- Asymptomatic (8/18)

Diagnosis and diagnostic procedures

- Earliest diagnosis made in the 5th GW, latest diagnosis in the 39th GW with the birth of a healthy neonate

- Diagnosed by ultrasound scan (11/18), exploratory laparotomy (5/18), MRI (1/18) or exploratory laparoscopy $(1 / 18)$

\section{Intervention}

- Surgical treatment with laparotomy (13/18), with additional administration of MTX in 2 cases

- Surgical treatment with laparoscopy (5/18), with additional administration of MTX in 1 case

\section{Placental management}

- Placental resection (15/18), combined with organ removal in 9 cases

- Placenta left in situ (3/18)
Some cases required a salpingectomy to remove the placenta and/or achieve hemostasis. It should be noted that some ectopic pregnancies were continued almost to term, with delivery of a live infant (6/18).

\section{C) Implantation in and on the liver ( $($ Table 3 )}

Presenting symptoms

- Abdominal pain (4/14), particularly in the right upper quadrant

- Abdominal pain radiating into the right shoulder (3/14)

- Abdominal pain and vaginal bleeding (2/14)

- Vaginal bleeding (1/14)

- Asymptomatic (3/14)

- Abdominal swelling (1/14)

\section{Diagnosis and diagnostic procedures}

- Earliest diagnosis made in the 5th GW, latest diagnosis in the 37 th GW with the birth of a healthy neonate

- Diagnosed by MRI (5/14), ultrasound scan (4/14), exploratory laparotomy (2/14), exploratory laparoscopy (1/14), CT (1/14) or PET-CT scan (1/14)

\section{Intervention}

- Surgical treatment with laparotomy (10/14), with additional administration of MTX in one case 
- Table 2 Implantation in and on the broad ligament of uterus.

\begin{tabular}{|c|c|c|c|c|c|c|c|c|c|c|}
\hline No. & Source & Age & GP score & GW & $\beta$-HCG & Symptoms & $\begin{array}{l}\text { Diagnostic } \\
\text { procedure }\end{array}$ & Intervention & $\begin{array}{l}\text { Placental } \\
\text { management }\end{array}$ & $\begin{array}{l}\text { Previous } \\
\text { medical } \\
\text { history }\end{array}$ \\
\hline 1 & $\begin{array}{l}\text { Abdul, 2008, } \\
\text { Nigeria [44] }\end{array}$ & 29 & G7P6 & 22 & - & $x$ & Sono & Ltm & $\begin{array}{l}\text { Resection, salpin- } \\
\text { go-oophorectomy }\end{array}$ & - \\
\hline 2 & $\begin{array}{l}\text { Abdul, 2008, } \\
\text { Nigeria [44] }\end{array}$ & 33 & G7P6 & 20 & - & $x$ & Sono & Ltm & $\begin{array}{l}\text { Resection, salpin- } \\
\text { go-oophorectomy }\end{array}$ & - \\
\hline 3 & $\begin{array}{l}\text { Akhtar, 2011, } \\
\text { Pakistan [45] }\end{array}$ & 35 & G3P2 & 37 & - & $A b P$ & Sono & $\begin{array}{l}\text { Ltm, live } \\
\text { birth, MTX }\end{array}$ & Left in situ & - \\
\hline 4 & $\begin{array}{l}\text { Atis, 2014, } \\
\text { Turkey [46] }\end{array}$ & 34 & Multipara & 8 & 10290 & LAbP, VB & Sono & Ltm & Resection & - \\
\hline 5 & $\begin{array}{l}\text { Cosentino, 2017, } \\
\text { Italy [47] }\end{array}$ & 35 & G3P1 & 12 & - & $x$ & Sono & Lap & $\begin{array}{l}\text { Resection, salpin- } \\
\text { go-oophorectomy }\end{array}$ & - \\
\hline 6 & $\begin{array}{l}\text { Dahab, 2011, } \\
\text { Saudi Arabia [48] }\end{array}$ & 23 & GOPO & 40 & 75542 & LAbP, Dys. & Sono & Ltm, live birth & Resection & - \\
\hline 7 & $\begin{array}{l}\text { Gudu, 2015, } \\
\text { Ethiopia [49] }\end{array}$ & 35 & P2 & 37 & - & $A b P, V B$ & Sono & Ltm, live birth & $\begin{array}{l}\text { Resection, } \\
\text { salpingectomy }\end{array}$ & - \\
\hline 8 & $\begin{array}{l}\text { Kar 2011, } \\
\text { India [50] }\end{array}$ & 31 & GOPO & 8 & 9470 & $x$ & Sono & MTX+ Lap & Resection & $\begin{array}{l}\text { IUI, endo- } \\
\text { metriosis }\end{array}$ \\
\hline 9 & $\begin{array}{l}\text { Kim, 2016, } \\
\text { Tanzania [51] }\end{array}$ & 27 & G3P2 & 13 & - & $A b P$ & MRI & Ltm & $\begin{array}{l}\text { Resection, } \\
\text { salpingo-oopho- } \\
\text { rectomy, partial } \\
\text { omentectomy }\end{array}$ & HIV positive \\
\hline 10 & $\begin{array}{l}\text { Nayar, 2016, } \\
\text { Portugal [52] }\end{array}$ & 25 & GOPO & 6 & 24719 & $A b P, V B$ & Sono & Lap & $\begin{array}{l}\text { Resection, } \\
\text { salpingectomy }\end{array}$ & \\
\hline 11 & $\begin{array}{l}\text { Parulekar, 2011, } \\
\text { India [53] }\end{array}$ & 22 & G2P1 & 5 & 1250 & LAbP & exLap & Lap & Resection & Lost IUD \\
\hline 12 & $\begin{array}{l}\text { Phupong, 2016, } \\
\text { Thailand [54] }\end{array}$ & 27 & G2P1 & 37 & - & $x$ & exLtm & Ltm, live birth & $\begin{array}{l}\text { Resection, hyster- } \\
\text { ectomy, salpingo- } \\
\text { oophorectomy }\end{array}$ & - \\
\hline 13 & $\begin{array}{l}\text { Rama, 2015, } \\
\text { India [55] }\end{array}$ & 23 & G2P1 & 12 & - & $\begin{array}{l}\text { AbP, VB, } \\
\text { Vom. }\end{array}$ & exLtm & Ltm & $\begin{array}{l}\text { Resection, salpin- } \\
\text { go-oophorectomy }\end{array}$ & - \\
\hline 14 & $\begin{array}{l}\text { Sassi, 2017, } \\
\text { Tunisia [56] }\end{array}$ & 32 & G2P1 & 5 & 26784 & LAbP & Sono & Lap & Resection & - \\
\hline 15 & $\begin{array}{l}\text { Seckin, 2011, } \\
\text { Turkey [57] }\end{array}$ & 28 & GOPO & 39 & - & $A b P$ & exLtm & Ltm, live birth & $\begin{array}{l}\text { Left in situ, re-Ltm } \\
\text { because of abscess } \\
\text { formation }\end{array}$ & - \\
\hline 16 & $\begin{array}{l}\text { Shamaash, 2017, } \\
\text { Egypt [58] }\end{array}$ & 25 & $\mathrm{G} 2 \mathrm{PO}+1$ & 17 & - & $x$ & exLtm & Ltm & $\begin{array}{l}\text { Resection, } \\
\text { salpingectomy }\end{array}$ & - \\
\hline 17 & $\begin{array}{l}\text { Sheethal, 2017, } \\
\text { India [59] }\end{array}$ & 28 & GOPO & 37 & - & $x$ & exLtm & Ltm, live birth & Resection & - \\
\hline 18 & $\begin{array}{l}\text { Yasutake, 2013, } \\
\text { Japan [60] }\end{array}$ & 34 & G2P1 & 8 & 13195 & $x$ & Sono & $\begin{array}{l}\text { Lap } \rightarrow \text { Ltm, } \\
\text { MTX }\end{array}$ & Left in situ & - \\
\hline
\end{tabular}

Abbreviations: $\mathrm{GP}$ score = gravidity and parity score, $\mathrm{GW}=$ week of gestation at diagnosis, $\beta$ - $\mathrm{HCG}$ in $\mathrm{mIU} / \mathrm{mL}$, Diagnostic procedure $=$ final procedure carried out to obtain the diagnosis, (L)AbP = (lower) abdominal pain, $\mathrm{VB}=$ vaginal bleeding, $\mathrm{X}=$ asymptomatic, Dys. $=$ dysuria, Vom. $=$ vomiting, exLap $/ \mathrm{Ltm}=$ exploratory laparoscopy/laparotomy, Sono = abdominal/transvaginal ultrasound scan, MRI = magnetic resonance imaging, Lap/Ltm = surgical treatment with laparoscopy/ laparotomy, MTX = methotrexate, IUI = intrauterine insemination, IUD = intrauterine device

- Surgical treatment with laparoscopy (2/14), with additional administration of MTX in both cases

- Feticide with potassium chloride (1/14) and administration of MTX

- Only administration of MTX (1/14)
Placental management

- Placental resection (7/14), combined with partial organ resection in 1 case

- Placenta left in situ (5/14)

- Not specified (2/14) 
- Table 3 Implantation in and on the liver.

\begin{tabular}{|c|c|c|c|c|c|c|c|c|c|c|}
\hline No. & Source & Age & GP score & GW & $\beta$-HCG & Symptoms & $\begin{array}{l}\text { Diagnostic } \\
\text { procedure }\end{array}$ & Intervention & $\begin{array}{l}\text { Placental } \\
\text { management }\end{array}$ & $\begin{array}{l}\text { Previous } \\
\text { medical } \\
\text { history }\end{array}$ \\
\hline 1 & $\begin{array}{l}\text { Brouard, 2015, } \\
\text { USA [61] }\end{array}$ & 20 & G4P4 & 37 & - & $x$ & exLtm & Ltm, live birth & Left in situ & - \\
\hline 2 & $\begin{array}{l}\text { Chin, 2010, } \\
\text { Singapore [62] }\end{array}$ & 30 & G2P1 & 5 & 1292 & $\begin{array}{l}\text { EgP, syn- } \\
\text { cope, RSP }\end{array}$ & exLap & Lap, MTX & Resection & - \\
\hline 3 & $\begin{array}{l}\text { Guo, 2016, } \\
\text { China [63] }\end{array}$ & 31 & - & - & 81418 & $\begin{array}{l}\text { abd. swell- } \\
\text { ing }\end{array}$ & MRI & Ltm & Resection & - \\
\hline 4 & $\begin{array}{l}\text { Hao, 2016, } \\
\text { China [64] }\end{array}$ & 31 & G2P1 & 6 & & $\begin{array}{l}\text { AbP, abd. } \\
\text { swelling }\end{array}$ & PET-CT & Ltm & - & - \\
\hline 5 & $\begin{array}{l}\text { Hu, 2014, } \\
\text { China [65] }\end{array}$ & 32 & G3P2 & 8 & & $\mathrm{EgP}$ & MRI & Ltm & - & - \\
\hline 6 & $\begin{array}{l}\text { Kuai, 2013, } \\
\text { China [66] }\end{array}$ & 33 & G4P2 & 6 & 186 & RUAP, RSP & MRI & Ltm & Resection & - \\
\hline 7 & $\begin{array}{l}\text { Ma, 2013, } \\
\text { China [67] }\end{array}$ & 31 & G6P2 & 8 & 23824 & RUAP & exLtm & Ltm, MTX & $\begin{array}{l}\text { Left in situ, } \\
\text { embolization }\end{array}$ & - \\
\hline 8 & $\begin{array}{l}\text { Moores, 2010*, } \\
\text { UK [68] }\end{array}$ & 23 & G1P0 & 12 & - & RUAP, RSP & Sono & $\mathrm{KCl}, \mathrm{MTX}$ & Left in situ & - \\
\hline 9 & $\begin{array}{l}\text { Qiao, 2013, } \\
\text { China [69] }\end{array}$ & 31 & G3P2 & 10 & 95700 & $x$ & MRI & Ltm & $\begin{array}{l}\text { Resection, } \\
\text { partial resection } \\
\text { of the liver }\end{array}$ & $\begin{array}{l}\text { Tubal } \\
\text { ligation }\end{array}$ \\
\hline 10 & $\begin{array}{l}\text { Ramphal, 2010, } \\
\text { South Africa [70] }\end{array}$ & 18 & - & 19 & - & $x$ & Sono & $\begin{array}{l}\text { Ltm in the } \\
34 \text { th GW, } \\
\text { live birth }\end{array}$ & Left in situ & - \\
\hline 11 & $\begin{array}{l}\text { Sibetcheu, 2017, } \\
\text { Cameroon [71] }\end{array}$ & 24 & G4P1 & 8 & 3000 & RUAP, VB & Sono & MTX & Left in situ & - \\
\hline 12 & $\begin{array}{l}\text { Wang, 2012, } \\
\text { Japan [72] }\end{array}$ & 33 & GOPO & 7 & 8988 & $A b P, V B$ & CT & Ltm & Resection & - \\
\hline 13 & $\begin{array}{l}\text { Yadav, 2012, } \\
\text { India [73] }\end{array}$ & 25 & G2P1 & 18 & & RUAP, Vom. & Sono & Ltm & $\begin{array}{l}\text { Resection, emboli- } \\
\text { zation, unsuccess- } \\
\text { ful hemostasis }\end{array}$ & - \\
\hline 14 & $\begin{array}{l}\text { Zhao, 2017, } \\
\text { China [74] }\end{array}$ & 21 & G0 & 14 & 135755 & VB & MRI & MTX, Lap & Resection & - \\
\hline
\end{tabular}

Abbreviations: $\mathrm{GP}$ score = gravidity and parity score, $\mathrm{GW}=$ week of gestation at diagnosis, $\beta$ - $\mathrm{HCG}$ in $\mathrm{mIU} / \mathrm{mL}$, Diagnostic procedure = final procedure carried out to obtain the diagnosis, ${ }^{*}=$ in the subhepatic space adjoining the gallbladder, (L)AbP = (lower) abdominal pain, EgP = epigastric pain, RUAP = right upper abdominal pain, RSP = right shoulder pain, VB = vaginal bleeding, Vom. = vomiting, exLap $/ \mathrm{Ltm}=$ exploratory laparoscopy/laparotomy, Sono = abdominal/transvaginal ultrasound scan, $\mathrm{CT}=$ computed tomography scan, $\mathrm{MRI}=$ magnetic resonance imaging, Lap $/ \mathrm{Ltm}=$ surgical treatment with laparoscopy/laparotomy

MRI and abdominal ultrasound were essential for diagnosis in those cases where the site of implantation was in the liver. In some cases, a live infant was delivered by laparotomy.

\section{D) Implantation on the greater omentum ( $>$ Table 4)}

Presenting symptoms

- Abdominal pain (10/12), often located in the lower abdomen, in one case with syncope

- Abdominal pain and vaginal bleeding with syncope (1/12)

- Not specified (1/12)
Diagnosis and diagnostic procedures

- Earliest diagnosis made in the 4th GW, latest diagnosis in the 14th GW

- Diagnosed by exploratory laparoscopy (5/12), ultrasound scan (4/12), MRI (1/12), exploratory laparotomy (1/12), or CT (1/ 12).

\section{Intervention}

- Surgical treatment with laparoscopy (7/12)

- Exploratory treatment with laparotomy (5/12) 
- Table 4 Implantation on the greater omentum.

\begin{tabular}{|c|c|c|c|c|c|c|c|c|c|c|}
\hline No. & Source & Age & GP score & GW & $\beta$-HCG & Symptoms & $\begin{array}{l}\text { Diagnostic } \\
\text { procedure }\end{array}$ & Intervention & $\begin{array}{l}\text { Placental } \\
\text { management }\end{array}$ & $\begin{array}{l}\text { Previous } \\
\text { medical } \\
\text { history }\end{array}$ \\
\hline 1 & $\begin{array}{l}\text { Allen, 2007, } \\
\text { UK [75] }\end{array}$ & 31 & $\mathrm{G} 2 \mathrm{P} 0+1$ & 14 & - & $\begin{array}{l}\text { EgP, Vom., } \\
\text { diarrhea }\end{array}$ & Sono & Ltm & Resection & Chlamydia \\
\hline 2 & $\begin{array}{l}\text { Bajis, 2018, } \\
\text { Australia [76] }\end{array}$ & 37 & G2P2 & 4 & 1480 & $\begin{array}{l}\text { LAbP, VB, } \\
\text { syncope }\end{array}$ & Sono & Lap $\times 2$ & Resection & - \\
\hline 3 & $\begin{array}{l}\text { Behjati, 2009, } \\
\text { UK [77] }\end{array}$ & 27 & G2P1 & - & 12709 & $\begin{array}{l}\text { LAbP, } \\
\text { syncope }\end{array}$ & exLap & Ltm & $\begin{array}{l}\text { Resection, partial } \\
\text { omentectomy }\end{array}$ & - \\
\hline 4 & $\begin{array}{l}\text { Chen, 2015, } \\
\text { China [78] }\end{array}$ & 18 & GOPO & 6 & 460 & LAbP & CT & Lap & $\begin{array}{l}\text { Resection, partial } \\
\text { omentectomy }\end{array}$ & - \\
\hline 5 & $\begin{array}{l}\text { Chopra, 2009, } \\
\text { India [79] }\end{array}$ & 29 & G4P3 & 6 & - & $A b P$ & Sono & Ltm & Resection & - \\
\hline 6 & $\begin{array}{l}\text { da Silva, 2008, } \\
\text { Brazil [80] }\end{array}$ & 36 & G3P3 & 13 & - & LAbP & exLtm & Ltm & $\begin{array}{l}\text { Resection, partial } \\
\text { omentectomy }\end{array}$ & - \\
\hline 7 & $\begin{array}{l}\text { Maiorana, 2014, } \\
\text { Italy [81] }\end{array}$ & 24 & G1P0 & 8 & 8047 & LAbP & exLap & Lap & Resection & - \\
\hline 8 & $\begin{array}{l}\text { Seol, 2010, } \\
\text { Korea [82] }\end{array}$ & 26 & G2P0 & - & - & LAbP & exLap & Lap & $\begin{array}{l}\text { Resection, partial } \\
\text { omentectomy }\end{array}$ & - \\
\hline 9 & $\begin{array}{l}\text { Srinivasan, 2014, } \\
\text { USA [83] }\end{array}$ & 20 & G2P1 & 8 & 1057 & LAbP & exLap & Lap & $\begin{array}{l}\text { Resection, partial } \\
\text { omentectomy }\end{array}$ & - \\
\hline 10 & $\begin{array}{l}\text { Takeda, 2016, } \\
\text { Japan [84] }\end{array}$ & 34 & G2P0 & 8 & - & - & MRI & Lap & $\begin{array}{l}\text { Resection, partial } \\
\text { omentectomy }\end{array}$ & \\
\hline 11 & $\begin{array}{l}\text { Tanase, 2013, } \\
\text { Japan [85] }\end{array}$ & 32 & G1P0 & 5 & - & LAbP & exLap & Lap & $\begin{array}{l}\text { Resection, partial } \\
\text { omentectomy }\end{array}$ & Pelv OP \\
\hline 12 & $\begin{array}{l}\text { Yip, 2016, } \\
\text { Singapore [86] }\end{array}$ & 31 & - & 6 & 11803 & EGP & Sono & Lap $\rightarrow$ Ltm & Resection & - \\
\hline
\end{tabular}

Abbreviations: $\mathrm{GP}$ score = gravidity and parity score, $\mathrm{GW}=$ week of gestation at diagnosis, $\beta$ - $\mathrm{HCG}$ in $\mathrm{mIU} / \mathrm{mL}$, Diagnostic procedure $=$ final procedure carried out to obtain the diagnosis, (L)AbP = (lower) abdominal pain, EgP = epigastric pain, $\mathrm{VB}=$ vaginal bleeding, Vom. = vomiting, exLap/Ltm = exploratory laparoscopy/ laparotomy, Sono = abdominal/transvaginal ultrasound scan, CT = computed tomography scan, MRI = magnetic resonance imaging, Lap $/ \mathrm{Ltm}=$ surgical treatment with laparoscopy/laparotomy, Pelv OP= prior history of pelvic surgery

\section{Placental management}

- Placental resection 12/12, combined with partial omentectomy in 7 cases.

\section{E) Implantation on the bowel and mesenteries $(\triangleright$ Table 5)}

Presenting symptoms

- Abdominal pain (4/9)

- Abdominal pain and vaginal bleeding (3/9)

- Hematochezia (1/9)

- Abdominal swelling (1/9)

\section{Diagnosis and diagnostic procedure}

- Earliest diagnosis made in the 6th GW, latest diagnosis in the 38th GW with the birth of a neonate

- Diagnosed by exploratory laparotomy (5/9), ultrasound scan (2/9), exploratory laparoscopy (1/9) or MRI (1/9).
Intervention

- Surgical treatment with laparotomy (8/9), with additional administration of MTX in two cases

- Surgical treatment with laparoscopy and administration of $\operatorname{MTX}(1 / 9)$

Placental management

- Placenta left in situ (5/9)

- Placental resection (4/9), with additional removal of organs in 3 cases

In the three cases who were delivered of a live infant, in addition to implanting on the bowel and its mesenteries, the placenta was also connected to the uterine serosa [11-13].

\section{F) Implantation on and in the spleen ( Table 6)}

Presenting symptoms

- Abdominal pain (5/9), particularly in the left upper abdomen

- Abdominal pain and vaginal bleeding (3/9)

- Asymptomatic (1/9) 
- Table 5 Implantation on the bowel and mesenteries.

\begin{tabular}{|c|c|c|c|c|c|c|c|c|c|c|}
\hline No. & Source & Age & GP score & GW & $\beta$-HCG & Symptoms & $\begin{array}{l}\text { Diagnostic } \\
\text { procedure }\end{array}$ & Intervention & $\begin{array}{l}\text { Placental } \\
\text { management }\end{array}$ & $\begin{array}{l}\text { Previous } \\
\text { medical } \\
\text { history }\end{array}$ \\
\hline 1 & $\begin{array}{l}\text { Anozie, 2016, } \\
\text { Nigeria [11] }\end{array}$ & 35 & G6P5 & $\begin{array}{l}\text { at } \\
\text { term }\end{array}$ & - & AbP, Resp. & Sono & $\begin{array}{l}\text { Ltm, live } \\
\text { birth, MTX }\end{array}$ & Left in situ & - \\
\hline 2 & $\begin{array}{l}\text { Baffoe, 2011, } \\
\text { Ghana [12] }\end{array}$ & 31 & G3P1 & 38 & - & $A b P, V B$ & exLtm & Ltm, live birth & Left in situ & - \\
\hline 3 & $\begin{array}{l}\text { Demendi, 2011, } \\
\text { Hungary [87] }\end{array}$ & 28 & G3P2 & 17 & & LAbP & Sono & Ltm, MTX & $\begin{array}{l}\text { Left in situ, } \\
\text { embolization }\end{array}$ & \\
\hline 4 & $\begin{array}{l}\text { Pichaichanlert, } \\
\text { 2017, Thailand } \\
{[88]}\end{array}$ & 32 & G1P1 & 15 & - & $\begin{array}{l}\text { blutiger } \\
\text { Stuhl }\end{array}$ & exLtm & Ltm & $\begin{array}{l}\text { Resection, partial } \\
\text { bowel resection, } \\
\text { re-anastomosis }\end{array}$ & Pelv OP, PID \\
\hline 5 & $\begin{array}{l}\text { Salathiel, 2016, } \\
\text { Chad [89] }\end{array}$ & 30 & G8L5 & 19 & - & AbP, VB & exLtm & Ltm & $\begin{array}{l}\text { Resection, } \\
\text { hysterectomy }\end{array}$ & - \\
\hline 6 & $\begin{array}{l}\text { Thompson, } \\
\text { 2011, UK [90] }\end{array}$ & 27 & - & 8 & - & LAbP & exLtm & Ltm & $\begin{array}{l}\text { Resection, } \\
\text { appendectomy }\end{array}$ & - \\
\hline 7 & $\begin{array}{l}\text { Tolefac, 2017, } \\
\text { Cameroon [13] }\end{array}$ & 22 & G3P0 & 25 & - & $\begin{array}{l}\text { abd. } \\
\text { swelling }\end{array}$ & exLtm & Ltm, live birth & Left in situ & \\
\hline 8 & $\begin{array}{l}\text { Trail, 2018, } \\
\text { UK [91] }\end{array}$ & 26 & G4P2 & 6 & 1647 & LAbP, SP & exLap & Lap, MTX & Left in situ & \\
\hline 9 & $\begin{array}{l}\text { Yildizhan, 2009, } \\
\text { Turkey [92] }\end{array}$ & 34 & G2P1 & 13 & - & AbP, BP, VB & MRI & Ltm & Resection & - \\
\hline
\end{tabular}

Abbreviations: $\mathrm{GP}$ score = gravidity and parity score, $\mathrm{GW}=$ week of gestation at diagnosis, $\beta$ - $\mathrm{HCG}$ in $\mathrm{mIU} / \mathrm{mL}$, Diagnostic procedure = final procedure carried out to obtain the diagnosis, (L)AbP = (lower) abdominal pain, $\mathrm{BP}=$ back pain, $\mathrm{SP}=$ shoulder pain, $\mathrm{VB}=$ vaginal bleeding, Resp. $=$ respiratory problems, exLap/ $\mathrm{Ltm}=$ exploratory laparoscopy/laparotomy, Sono = abdominal/transvaginal ultrasound scan, MRI = magnetic resonance imaging, Lap $/ \mathrm{Ltm}=$ surgical treatment with laparoscopy/laparotomy, Pelv OP = prior history of pelvic surgery, PID = pelvic inflammatory disease

- Table 6 Implantation on and in the spleen.

\begin{tabular}{|c|c|c|c|c|c|c|c|c|c|c|}
\hline No. & Source & Age & GP score & GW & $\beta$-HCG & Symptoms & $\begin{array}{l}\text { Diagnostic } \\
\text { procedure }\end{array}$ & Intervention & $\begin{array}{l}\text { Placental } \\
\text { management }\end{array}$ & $\begin{array}{l}\text { Previous } \\
\text { medical } \\
\text { history }\end{array}$ \\
\hline 1 & $\begin{array}{l}\text { Biolchini, 2010, } \\
\text { Italy [93] }\end{array}$ & 41 & G3P- & 4 & 8980 & LUAP & CT & Lap & $\begin{array}{l}\text { Resection, } \\
\text { splenectomy }\end{array}$ & - \\
\hline 2 & $\begin{array}{l}\text { Gao, 2017, } \\
\text { China [94] }\end{array}$ & 27 & GOPO & 8 & 119027 & $\begin{array}{l}\text { AbP, VB, } \\
\text { dizziness }\end{array}$ & Sono & Ltm & $\begin{array}{l}\text { Resection, } \\
\text { splenectomy }\end{array}$ & - \\
\hline 3 & $\begin{array}{l}\text { Greenbaum, } \\
\text { 2016, USA [95] }\end{array}$ & 27 & G2P2 & 4 & 1865 & LUAP, Vom. & exLap & Ltm & $\begin{array}{l}\text { Resection, } \\
\text { splenectomy }\end{array}$ & - \\
\hline 4 & $\begin{array}{l}\text { Klang, 2016, } \\
\text { Israel [96] }\end{array}$ & 35 & G3P1 & - & 71000 & $x$ & CT & $\mathrm{KCl}, \mathrm{MTX}$ & Left in situ & GA, PCOS \\
\hline 5 & $\begin{array}{l}\text { Perez 2008*, } \\
\text { USA [97] }\end{array}$ & 36 & G1P0 & - & - & LAbP & exLap & Lap & Resection & $\begin{array}{l}\text { Unicornuate } \\
\text { uterus, renal } \\
\text { agenesis }\end{array}$ \\
\hline 6 & $\begin{array}{l}\text { Python, 2016, } \\
\text { USA [98] }\end{array}$ & 21 & G1 & 5 & 8476 & $A b P, V B$ & CT & MTX & Left in situ & - \\
\hline 7 & $\begin{array}{l}\text { Rathore, 2017, } \\
\text { Turkey [99] }\end{array}$ & 23 & G1P1 & 4 & 6565 & LUAP, Vom. & exLtm & Ltm & $\begin{array}{l}\text { Resection, } \\
\text { splenectomy }\end{array}$ & - \\
\hline 8 & $\begin{array}{l}\text { Wu, 2017, } \\
\text { Japan [100] }\end{array}$ & 27 & G1P0 & 8 & 119027 & AbP & Sono & Ltm & $\begin{array}{l}\text { Resection, } \\
\text { splenectomy }\end{array}$ & - \\
\hline 9 & $\begin{array}{l}\text { Wu, 2018, } \\
\text { China [101] }\end{array}$ & 29 & G3P2 & 8 & 16669 & $\mathrm{VB}, \mathrm{AbP}$ & Sono & Ltm & $\begin{array}{l}\text { Resection, } \\
\text { splenectomy }\end{array}$ & \\
\hline
\end{tabular}

Abbreviations: $\mathrm{GP}$ score = gravidity and parity score, $\mathrm{GW}=$ week of gestation at diagnosis, $\beta$ - $\mathrm{HCG}$ in $\mathrm{mIU} / \mathrm{mL}$, Diagnostic procedure $=$ final procedure carried out to obtain the diagnosis, ${ }^{*}=$ subsplenic, $(\mathrm{L}) \mathrm{AbP}=($ lower) abdominal pain, $\mathrm{LUAP}=$ left upper abdominal pain, $\mathrm{VB}=$ vaginal bleeding, Vom. $=$ vomiting, $\mathrm{X}=$ asymptomatic, exLap/Ltm = exploratory laparoscopy/laparotomy, Sono = abdominal/transvaginal ultrasound scan, CT = computed tomography scan, $\mathrm{Lap} / \mathrm{Ltm}=$ surgical treatment with laparoscopy/laparotomy, MTX = methotrexate, GA = gonadotropin analogs, PCOS= polycystic ovary syndrome 
- Table 7 Other rare intraperitoneal implantation sites.

\begin{tabular}{|c|c|c|c|c|c|c|c|c|c|c|}
\hline No. & Source & Age & GP score & GW & $\beta$-HCG & Symptoms & $\begin{array}{l}\text { Diagnostic } \\
\text { procedure }\end{array}$ & Intervention & $\begin{array}{l}\text { Placental } \\
\text { management }\end{array}$ & $\begin{array}{l}\text { Previous } \\
\text { medical } \\
\text { history }\end{array}$ \\
\hline \multicolumn{11}{|c|}{ Abdominal wall } \\
\hline 1 & $\begin{array}{l}\text { Anderson, 2009, } \\
\text { USA [102] }\end{array}$ & 26 & G4P1 & 3 & 8979 & $A b P, V B$ & CT & MTX & Left in situ & - \\
\hline 2 & $\begin{array}{l}\text { Borton, 2015, } \\
\text { UK [103] }\end{array}$ & 38 & G2P0 & 7 & 2208 & $A b P, V B$ & exLap & MTX & Left in situ & $\begin{array}{l}\text { PID, endo- } \\
\text { metriosis }\end{array}$ \\
\hline 3 & $\begin{array}{l}\text { Gorry, 2012, } \\
\text { UK [104] }\end{array}$ & 32 & & 8 & - & $A b P, V B$ & exLap & Lap & Left in situ & - \\
\hline 4 & $\begin{array}{l}\text { Irani, 2016, } \\
\text { USA [105] }\end{array}$ & 36 & GOPO & 4 & 998 & $A b P$ & exLap & Lap & Resection & IVF \\
\hline 5 & $\begin{array}{l}\text { Lee, 2015, } \\
\text { Cameroon [106] }\end{array}$ & 21 & G2P2 & 36 & - & LAbP & Sono & Ltm, MTX & $\begin{array}{l}\text { Left in situ, re-Ltm } \\
\text { because of abscess } \\
\text { formation }\end{array}$ & - \\
\hline \multicolumn{11}{|c|}{ Diaphragm } \\
\hline 1 & $\begin{array}{l}\text { Chen, 2019, } \\
\text { China [107] }\end{array}$ & 33 & G-P1 & 12 & 3129 & RUAP, RSP & CT & Lap & Resection & - \\
\hline \multicolumn{11}{|c|}{ Inguinal canal } \\
\hline 1 & $\begin{array}{l}\text { Noguchi, 2014, } \\
\text { Japan [108] }\end{array}$ & 45 & G5P4 & 8 & 3090 & $\begin{array}{l}\text { Swelling } \\
\text { and pain } \\
\text { in the right } \\
\text { groin }\end{array}$ & Sono & $\mathrm{Ltm}$ & Resection & $\begin{array}{l}\text { Endo- } \\
\text { metriosis }\end{array}$ \\
\hline
\end{tabular}

Abbreviations: GP score = gravidity and parity score, $\mathrm{GW}=$ week of gestation at diagnosis, $\beta$ - $\mathrm{HCG}$ in $\mathrm{mIU} / \mathrm{mL}$, Diagnostic procedure = final procedure carried out to obtain the diagnosis, (L)AbP = (lower) abdominal pain, RUAP = right upper abdominal pain, $\mathrm{RSP}=$ right shoulder pain, $\mathrm{VB}=$ vaginal bleeding, exLap $/ \mathrm{Ltm}=$ exploratory laparoscopy/laparotomy, Sono = abdominal/transvaginal ultrasound scan, CT = computed tomography scan, Lap/Ltm = surgical treatment with laparoscopy/laparotomy, MTX = methotrexate

\section{Diagnosis and diagnostic procedures}

- Earliest diagnosis made in the 4th GW, latest diagnosis in the 8th GW

- Diagnosed by ultrasound scan (3/9), CT scan (3/9), exploratory laparoscopy (2/9), or exploratory laparotomy (1/9)

Intervention

- Surgical treatment with laparotomy (5/9)

- Surgical treatment with laparoscopy (2/9)

- Feticide with potassium chloride and administration of MTX $(1 / 9)$

- Only administration of MTX (1/9)

Placental management

- Placental resection (7/9), combined with splenectomy in 6 cases

- Placenta left in situ (2/9)

\section{G) Implantation on the peritoneum of the abdominal/} pelvic wall ( $>$ Table 7 )

Presenting symptoms

- Abdominal pain (2/5)

- Abdominal pain and vaginal bleeding (3/5)

\section{Diagnosis and diagnostic procedures}

- Earliest diagnosis made in the 3rd GW, latest diagnosis in the 36th GW with the delivery of a dead infant

- Diagnosed by exploratory laparoscopy (3/5), ultrasound scan $(1 / 5)$ or CT scan $(1 / 5)$

Treatment

- Surgical treatment with laparoscopy (2/5)

- Surgical treatment with laparotomy and the administration of $\operatorname{MTX}(1 / 5)$

- In 2 cases only administration of MTX

Placental management

- Placenta left in situ (4/5); one of these cases required re-laparotomy because of abscess formation

- Placental resection (1/5)

\section{$\mathrm{H})$ Implantation in the paraaortic/paracaval} retroperitoneal space ( $>$ Table 8 )

Presenting symptoms

- Abdominal pain (4/7)

- Abdominal pain and vaginal bleeding (1/7)

- Asymptomatic (2/7) 
- Table 8 Extraperitoneal implantation sites.

\begin{tabular}{|c|c|c|c|c|c|c|c|c|c|c|}
\hline No. & Source & Age & GP score & GW & $\beta$-HCG & Symptoms & $\begin{array}{l}\text { Diagnostic } \\
\text { procedure }\end{array}$ & Intervention & $\begin{array}{l}\text { Placental } \\
\text { management }\end{array}$ & $\begin{array}{l}\text { Previous } \\
\text { medical } \\
\text { history }\end{array}$ \\
\hline \multicolumn{11}{|c|}{ Paraaortic and paracaval } \\
\hline 1 & $\begin{array}{l}\text { Iwama, 2008, } \\
\text { Japan [109] }\end{array}$ & 31 & G1P0 & 10 & 45369 & $\mathrm{AbP}$ & MRI & MTX, Ltm & Resection & $\begin{array}{l}\text { EP, IVF, sal- } \\
\text { pingectomy }\end{array}$ \\
\hline 2 & $\begin{array}{l}\text { Jiang, 2014, } \\
\text { China [110] }\end{array}$ & 33 & G3P2 & 7 & - & $\mathrm{AbP}$ & MRI & MTX, Ltm & Resection & \\
\hline 3 & $\begin{array}{l}\text { Ouassour, 2017, } \\
\text { Morocco [111] }\end{array}$ & 35 & G4P2 & 7 & 29386 & $x$ & Sono & Ltm & Resection & $\begin{array}{l}\text { EP, salpin- } \\
\text { gectomy }\end{array}$ \\
\hline 4 & $\begin{array}{l}\text { Pak, 2018, } \\
\text { USA [112] }\end{array}$ & 30 & G4P3 & 8 & 40532 & $\mathrm{AbP}$ & exLtm & $\mathrm{Ltm}$ & Resection & - \\
\hline 5 & $\begin{array}{l}\text { Wang, 2017, } \\
\text { China [9] }\end{array}$ & 32 & G4P1 & 5 & 10652 & $x$ & CT & Lap & Resection & $\begin{array}{l}\text { Salpin- } \\
\text { gectomy }\end{array}$ \\
\hline 6 & $\begin{array}{l}\text { Yang, 2018, } \\
\text { China [113] }\end{array}$ & 34 & G2P0 & 7 & 6803 & $A b P, V B$ & CT & Ltm & Resection & - \\
\hline 7 & $\begin{array}{l}\text { Zhang, 2018, } \\
\text { China [114] }\end{array}$ & 29 & & 9 & 16453 & LAbP & Sono & $\begin{array}{l}\text { Emb., MTX, } \\
\text { Ltm }\end{array}$ & Resection & - \\
\hline \multicolumn{11}{|c|}{ Rectovaginal space } \\
\hline 1 & $\begin{array}{l}\text { Martinez, 2011, } \\
\text { Spain [115] }\end{array}$ & 37 & G2P1 & $\begin{array}{l}29 \text { days } \\
\text { after IUI }\end{array}$ & 7787 & LAbP & Sono & Lap, MTX & Resection & IUI \\
\hline 2 & $\begin{array}{l}\text { Yang, 2017, } \\
\text { China [116] }\end{array}$ & 32 & G5P1 & 6 & 1880 & LAbP & exLap & Lap & Resection & - \\
\hline \multicolumn{11}{|c|}{ Obturator foramen } \\
\hline 1 & $\begin{array}{l}\text { Lin, 2008, } \\
\text { China [117] }\end{array}$ & 19 & G1P0 & 7 & 267 & LAbP, VB & exLap & $\mathrm{Ltm}$ & Resection & \\
\hline 2 & $\begin{array}{l}\text { Persson, 2010, } \\
\text { Sweden [10] }\end{array}$ & 33 & G3P1 & $\begin{array}{l}27 \text { days } \\
\text { after ET }\end{array}$ & 18032 & VB & Sono & Lap $\times 2$ & Resection & EP, ET \\
\hline \multicolumn{11}{|c|}{ Renal fascia } \\
\hline 1 & $\begin{array}{l}\text { Chishima, 2013, } \\
\text { Japan [118] }\end{array}$ & 33 & G3P2 & 7 & 3100 & $\mathrm{AbP}$ & CT & Ltm & Resection & - \\
\hline \multicolumn{11}{|c|}{ Pancreas } \\
\hline 1 & $\begin{array}{l}\text { Guan, 2015, } \\
\text { China [119] }\end{array}$ & 30 & G1P0 & 5 & 2500 & $\mathrm{EgP}$ & MRI & MTX, Lap & $\begin{array}{l}\text { Resection pan- } \\
\text { createctomy, } \\
\text { splenectomy }\end{array}$ & - \\
\hline \multicolumn{11}{|c|}{ Paravesical space } \\
\hline 1 & $\begin{array}{l}\text { Meire, 2007, } \\
\text { Netherlands } \\
{[120]}\end{array}$ & 30 & G3P1 & 20 & - & LAbP & exLtm & MTX, Ltm & Resection & - \\
\hline
\end{tabular}

\section{Diagnosis and diagnostic procedures}

- Earliest diagnosis made in the 5th $\mathrm{GW}$, latest diagnosis in the 10th GW

- Diagnosed by ultrasound scan (2/7), CT scan (2/7), MRI (2/7), or exploratory laparotomy $(1 / 7)$

Treatment

- Surgical treatment with laparotomy (6/7), with additional administration of MTX in 3 cases

- Surgical treatment with laparoscopy (1/7)

\section{Placental management}

- Placental resection (7/7)

Implantation sites of retroperitoneal EP were often found in the paraaortic, paracaval or paravesicular space and usually required surgical resection by laparotomy. 


\section{Discussion}

A total of 115 case reports from 113 publications were analyzed for this literature review. The most commonly reported EP implantation sites were the uterine serosa (32/115 or $27.8 \%$ ), the broad ligament of uterus ( $18 / 115$ or $15.7 \%)$, the liver $(14 / 115$ or $12.2 \%)$ and the greater omentum (12/115 or $10.4 \%)$. Other possible locations for peritoneal EP were the serosa of the bowel, spleen or abdominal wall. There are reports of individual cases with implantation on the diaphragm [107] and in the inguinal canal [108]. A number of retroperitoneal EP sites were found, particularly in the paraaortic and paracaval space but also in the rectovaginal space, on the obturator foramen, in the paravesical space or in the vicinity of the pancreas or kidney.

As regards rare EP implantation sites, their symptoms and the diagnostic procedures used to determine EP, these data largely correspond to the information collated by Molinaro and Barnhart [6]. However, the data on EP implantation sites in the liver and in the retroperitoneal space were not yet available to Molinaro and Barnhart [6] and have been added here. Various abdominal implantation sites were briefly cited in the above-mentioned review by Parker and Srinivas [3]. Our review added a summary of a number of additional case reports to their more limited data, including reports covering implantation on the diaphragm, in the inguinal canal, at the obturator foramen, on a renal capsule and on the pancreas.

For clinical practice, the symptoms of the affected patient are crucial. The most commonly reported symptom was abdominal pain, with fewer reports of vaginal bleeding. However, there are also a number of reports of abdominal ectopic pregnancies occurring in asymptomatic patients, with a total of 24/115 (20.9\%) patients in our review reporting no symptoms. Abdominal symptoms and a $\beta$-HCG serum value of more than $1500 \mathrm{mU} / \mathrm{mL}$ with no intrauterine pregnancy visible on imaging are indications for an EP and must be immediately investigated further with additional diagnostic procedures or by exploratory laparoscopy [93]. The importance of carrying out laparoscopic inspection of both the lower and the upper abdominal region must be emphasized (comprehensive inspection) [98]. In the case reports which we found during our search, the most common intervention was surgical resection by laparotomy, particularly in cases of advanced pregnancy, surgery in the retroperitoneal space, and in countries with more limited medical resources. However, there are also reports of the successful management of specific cases of EP in rare locations [52,62, 85, 93].

The appropriate strategy for placental management is disputed in the literature and decisions must be made on a case-bycase basis according to the individual implantation site, the risk of bleeding, the patient's clinical condition, the physician's surgical experience and the available medical resources [28, 62]. Implantations on the omentum, spleen or liver are associated with a higher risk of bleeding, while the risk of bleeding appears to be lower if the placenta is located on the uterine serosa [37]. According to some authors, the extent of placental adhesion can be determined preoperatively using MRI [121]. Provided that placental blood supply can be safely disrupted, then resection of the placenta is recommended $[37,40,57]$. Otherwise the placenta can be left in situ, after the umbilical cord and fetal membranes have been removed [4]. A postoperative course of antibiotics to prevent infection combined with abdominal drainage to detect bleeding are recommended [87]. Imaging procedures and changes in $\beta$-HCG values can be used to monitor resorption of the placenta [22, 23, 30]. Varying doses of methotrexate were administered in some cases to hasten trophoblast degeneration [18, $31,67,87]$.

Reports of an abdominal EP resulting in the birth of a viable infant are rare in the literature. Their prevalence is estimated to be less than $0.01 \%$ of all hospital births [30]. Many authors refer to pregnancies after the 20th GW as advanced abdominal pregnancies; it has been suggested that conservative management could be considered in these cases after weighing up the maternal risk of bleeding [37]. In our case series, the majority of live infants were delivered to patients with placental implantation on the external serosa of the uterine wall. This was also observed by Rohilla et al. in her review of advanced abdominal pregnancies [37]. Individual cases who underwent planned conservative management of an abdominal EP with elective laparotomy and the birth of a healthy infant in the 34th GW have been described $[25,70]$.

\section{Conclusion}

This literature review makes it clear that in the event of a positive pregnancy test, an "empty" uterus, and abdominal pain with or without vaginal bleeding in women of childbearing age, it is important not only to investigate for tubal pregnancy as the most common ectopic pregnancy site but also to consider the possibility of ectopic pregnancies in rarer ectopic sites. At the same time, the relatively high percentage of asymptomatic patients and the often very late diagnosis highlight the importance of obtaining a detailed abdominal vaginal ultrasound scan. Abdominal CT or MRI imaging may provide additional useful information. While this is available in highly developed industrialized countries, CT imaging and MRI can be a problem in countries with more limited healthcare resources [13]. MRI is the best method of obtaining a diagnosis and planning the treatment of an EP located in the intra- or extraperitoneal space $[3,6,65,110]$. The appropriate placental management strategy and the possibility of achieving a live birth must always be considered on case-by-case basis and weighed up after carefully assessing the maternal morbidity risk.

Future studies on rare EP locations should focus on previously unknown risk factors in this group of EP patients, using retrospective case-control studies. The pathogenesis of retroperitoneal implantation of ectopic pregnancy is still unknown and also merits further study.

\section{Conflict of Interest}

The authors declare that they have no conflict of interest. 


\section{References}

[1] National Institute for Health and Care Excellence. Ectopic pregnancy and miscarriage: diagnosis and initial management 2019. Online: https:// www.nice.org.uk/guidance/ng126; last access: 30.05.2019

[2] Cecchino GN, Araujo Junior E, Elito Junior J. Methotrexate for ectopic pregnancy: when and how. Arch Gynecol Obstet 2014; 290: 417-423. doi:10.1007/s00404-014-3266-9

[3] Parker VL, Srinivas M. Non-tubal ectopic pregnancy. Arch Gynecol Obstet 2016; 294: 19-27. doi:10.1007/s00404-016-4069-y

[4] Hailu FG, Yihunie GT, Essa AA et al. Advanced abdominal pregnancy, with live fetus and severe preeclampsia, case report. BMC Pregnancy Childbirth 2017; 17: 243. doi:10.1186/s12884-017-1437-y

[5] Miyauchi A, Yamada M, Furuya M et al. Peritoneal pregnancy with massive hemoperitoneum in early gestation: two case reports. Clin Case Rep 2015; 3: 431-437. doi:10.1002/ccr3.266

[6] Molinaro TA, Barnhart KT. Ectopic pregnancies in unusual locations. Semin Reprod Med 2007; 25: 123-130. doi:10.1055/s-2007-970051

[7] Arbeitsgemeinschaft der wissenschaftlichen Medizinischen Fachgemeinschaften (AWMF). Angemeldetes Leitlinienvorhaben: Extrauteringravidität (17.01.2019). Online: https://www.awmf.org/leitlinien/ detail/anmeldung/1/II/015-076.html; last access: 30.05.2019

[8] Shen L, Fu J, Huang W et al.; Gynaecology and Fertility Group. Interventions for non-tubal ectopic pregnancy. Online Protocol. Cochrane Database Syst Rev 2014. doi:10.1002/14651858.CD011174

[9] Wang L, Tong X, Li H et al. Case Report An early extra peritoneal ectopic pregnancy successfully treated with laparoscopy: a case report. Int J Clin Exp Med 2017; 10: 3855-3859

[10] Persson J, Reynisson P, Masback A et al. Histopathology indicates lymphatic spread of a pelvic retroperitoneal ectopic pregnancy removed by robot-assisted laparoscopy with temporary occlusion of the blood supply. Acta Obstet Gynecol Scand 2010; 89: 835-839. doi:10.3109/ 00016341003623779

[11] Anozie OB, Esike CU, Eze JN et al. Advanced abdominal ectopic pregnancy: a case report. Open J Obstet Gynecol 2016; 6: 360

[12] Baffoe P, Fofie C, Gandau BN. Term abdominal pregnancy with healthy newborn: a case report. Ghana Med J 2011; 45: 81-83

[13] Tolefac PN, Abanda MH, Minkande JZ et al. The challenge in the diagnosis and management of an advanced abdominal pregnancy in a resource-low setting: a case report. J Med Case Rep 2017; 11: 199. doi:10.1186/s13256-017-1369-1

[14] Creinin MD, Simhan HN. Can we communicate gravidity and parity better? Obstet Gynecol 2009; 113: 709-711. doi:10.1097/AOG.0b013e $3181988 f 8 f$

[15] Abdelrahman S, Deeter M, Muthusami A et al. A live term intra-abdominal pregnancy in a Field Hospital: a case report. J Surg Case Rep 2017; 2017: rjx062. doi:10.1093/jscr/rjx062

[16] Bhoil R, Aggarwal N, Jhobta A et al. Advanced abdominal pregnancy with successful outcome. Intern Emerg Med 2016; 11: 877-878. doi:10.1007/s11739-015-1339-9

[17] Bohiltea R, Radoi V, Tufan C et al. Abdominal pregnancy - Case presentation. J Med Life 2015; 8: 49-54

[18] Cho FN, Chen SN, Chang YH et al. Alternative management of a sizable cul-de-sac ectopic pregnancy. Taiwan J Obstet Gynecol 2015; 54: $206-$ 207. doi:10.1016/j.tjog.2014.10.006

[19] Dabiri T, Marroquin GA, Bendek B et al. Advanced extrauterine pregnancy at 33 weeks with a healthy newborn. Biomed Res Int 2014; 2014: 102479. doi:10.1155/2014/102479

[20] Dassah ET, Odoi AT, Opoku BK. Advanced twin abdominal pregnancy: diagnostic and therapeutic challenges. Acta Obstet Gynecol Scand 2009; 88: 1291-1293. doi:10.3109/00016340903281006
[21] Gayer G. Images in clinical medicine. Abdominal ectopic pregnancy. N Engl J Med 2012; 367: 2334. doi:10.1056/NEJMicm1111814

[22] Gidiri MF, Kanyenze M. Advanced abdominal ectopic pregnancy: lessons from three cases from Zimbabwe and a literature appraisal of diagnostic and management challenges. Womens Health (Lond) 2015; 11: 275279. doi:10.2217/whe. 15.3

[23] Hishikawa K, Fukuda T, Inoue $\mathrm{H}$ et al. Laparoscopic Management of Abdominal Pregnancy with Local Injection of Vasopressin Solution: A Case Report. Am J Case Rep 2016; 17: 637-640. doi:10.12659/ajcr.898554

[24] Isah AY, Ahmed Y, Nwobodo El et al. Abdominal pregnancy with a full term live fetus: case report. Ann Afr Med 2008; 7: 198-199

[25] Kim MJ, Bae JY, Seong W] et al. Sonographic diagnosis of a viable abdominal pregnancy with planned delivery after fetal lung maturation. J Clin Ultrasound 2013; 41: 563-565. doi:10.1002/jcu.22010

[26] Marcelin C, Kouchner P, Bintner M et al. Placenta embolization of advanced abdominal pregnancy. Diagn Interv Imaging 2018; 99: 265266. doi:10.1016/j.diii.2017.12.001

[27] Mengistu Z, Getachew A, Adefris M. Term abdominal pregnancy: a case report. J Med Case Rep 2015; 9: 168. doi:10.1186/s13256-015-0635-3

[28] Muehlparzer L, Arzt W, Ebner T et al. Secondary abdominal pregnancy with live birth. Acta Obstet Gynecol Scand 2011; 90: 288. doi:10.1111/ j.1600-0412.2010.01041.x

[29] Nassali MN, Benti TM, Bandani-Ntsabele M et al. A case report of an asymptomatic late term abdominal pregnancy with a live birth at 41 weeks of gestation. BMC Res Notes 2016; 9: 31. doi:10.1186/s13104016-1844-6

[30] Nkusu Nunyalulendho D, Einterz EM. Advanced abdominal pregnancy: case report and review of 163 cases reported since 1946. Rural Remote Health 2008; 8: 1087

[31] Panagiotakis A, Brown M, Asulin Y et al. A Case Report of Successful Treatment of an Abdominal Ectopic Pregnancy with Methotrexate. J Minim Invasive Gynecol 2009; 16: S126

[32] Parekh VK, Bhatt S, Dogra VS. Abdominal pregnancy: an unusual presentation. J Ultrasound Med 2008; 27: 679-681

[33] Patel C, Feldman J, Ogedegbe C. Complicated abdominal pregnancy with placenta feeding off sacral plexus and subsequent multiple ectopic pregnancies during a 4-year follow-up: a case report. J Med Case Rep 2016; 10: 37. doi:10.1186/s13256-016-0808-8

[34] Pieh-Holder KL, Scardo JA, Costello DH. Lactogenesis failure following successful delivery of advanced abdominal pregnancy. Breastfeed Med 2012; 7: 543-546. doi:10.1089/bfm.2011.0131

[35] Pradhan M, Sreenivas M, Singh B et al. Sudden death in advanced abdominal pregnancy: a case report and discussion of the related medicolegal issues. Med Sci Law 2013; 53: 90-92. doi:10.1258/msl.2012. 012014

[36] Promsonthi P, Herabutya Y. Uterocutaneous fistula in term abdominal pregnancy. Eur J Obstet Gynecol Reprod Biol 2007; 132: 239-241. doi:10.1016/j.ejogrb.2006.04.041

[37] Rohilla M, Joshi B, Jain $V$ et al. Advanced abdominal pregnancy: a search for consensus. Review of literature along with case report. Arch Gynecol Obstet 2018; 298: 1-8. doi:10.1007/s00404-018-4743-3

[38] Sib SR, Ouedraogo I, Sanogo M et al. A full term abdominal pregnancy with an isthmic tubal implantation of the placenta. BMC Pregnancy Childbirth 2018; 18: 448. doi:10.1186/s12884-018-2071-z

[39] Shih CC, Lee RK, Hwu YM. Cul-de-sac pregnancy following in vitro fertilization and embryo transfer. Taiwan J Obstet Gynecol 2007; 46: 171173. doi:10.1016/s1028-4559(07)60013-8

[40] Tucker K, Bhardwaj NR, Clark E et al. Delayed diagnosis and management of second trimester abdominal pregnancy. BMJ Case Rep 2017; 2017: bcr2017221433. doi:10.1136/bcr-2017-221433 
[41] Yanaihara A, Ohgi S, Motomura K et al. An abdominal ectopic pregnancy following a frozen-thawed ART cycle: a case report and review of the literature. BMC Pregnancy Childbirth 2017; 17: 108. doi:10.1186/s12884017-1294-8

[42] Yoder N, Tal R, Martin JR. Abdominal ectopic pregnancy after in vitro fertilization and single embryo transfer: a case report and systematic review. Reprod Biol Endocrinol 2016; 14: 69. doi:10.1186/s12958-0160201-x

[43] Zhang J, Li F, Sheng Q. Full-term abdominal pregnancy: a case report and review of the literature. Gynecol Obstet Invest 2008; 65: 139-141. doi:10.1159/000110015

[44] Abdul MA, Tabari AM, Kabiru D et al. Broad ligament pregnancy: a report of two cases. Ann Afr Med 2008; 7: 86-87

[45] Akhtar N. Ectopic pregnancy and full term live birth: a case report. J Postgrad Med Inst 2011; 25: 181-182

[46] Atis A, Gunduz O, Tolga Karacan KA. A Rare Form of Ectopic Pregnancy on Mesoovarium, Case Report. J Clin Case Rep 2014; 4: 359

[47] Cosentino F, Rossitto C, Turco LC et al. Laparoscopic Management of Abdominal Pregnancy. J Minim Invasive Gynecol 2017; 24: 724-725. doi:10.1016/j.jmig.2017.01.023

[48] Dahab AA, Aburass R, Shawkat W et al. Full-term extrauterine abdominal pregnancy: a case report. J Med Case Rep 2011; 5: 531. doi:10.1186/ $1752-1947-5-531$

[49] Gudu W, Bekele D. A pre-operatively diagnosed advanced abdominal pregnancy with a surviving neonate: a case report. J Med Case Rep 2015; 9: 228. doi:10.1186/s13256-015-0712-7

[50] Kar S. Primary abdominal pregnancy following intra-uterine insemination. J Hum Reprod Sci 2011; 4: 95-99. doi:10.4103/0974-1208.86091

[51] Kim MC, Manyanga H, Lwakatare F. Secondary abdominal pregnancy in human immunodeficiency virus-positive woman. Obstet Gynecol Sci 2016; 59: 535-538. doi:10.5468/ogs.2016.59.6.535

[52] Nayar J, Nair SS. Broad Ligament Pregnancy - Success Story of a Laparoscopically Managed Case. J Clin Diagn Res 2016; 10: Qd04-QD05. doi:10.7860/jcdr/2016/19293.8136

[53] Parulekar SV. Face to face: misplaced intrauterine device and abdominal ectopic pregnancy. J Postgrad Med 2011; 57: 223-224. doi:10.4103/ 0022-3859.85217

[54] Phupong V, Uthaisan J, Tantbirojn P. Undiagnosed term intraligamentous pregnancy: A case report and literature review. J Obstet Gynaecol 2016; 36: 432-434. doi:10.3109/01443615.2015.1101432

[55] Rama C, Lepakshi G, Raju SN. Broad Ligament ectopic pregnancy. Journal of Clinical and Scientific Research 2015; 4: 45-48. doi:10.15380/22775706.JCSR. 14.013

[56] Sassi A, Dimassi K, Ben Slama S et al. A broad ligament pregnancy successfully managed by laparoscopy. J Obstet Gynaecol 2018; 38: 423424. doi:10.1080/01443615.2017.1342231

[57] Seckin B, Turkcapar FA, Tarhan I et al. Advanced intraligamentary pregnancy resulting in a live birth. J Obstet Gynaecol 2011; 31: 260-261. doi:10.3109/01443615.2011.553691

[58] Shamaash AH, Abbas AM. Undiagnosed asymptomatic second trimester broad ligament ectopic pregnancy: a case report and mini-review. Proc Obstet Gynecol 2017; 7: 1-8

[59] Sheethal $\mathrm{CH}$, Powar A. Full term viable secondary broad ligament pregnancy - A rare case. Case Rep Womens Health 2017; 13: 4-5. doi:10.1016/j.crwh.2016.10.003

[60] Yasutake N, Tsunematsu R, Oishi H et al. Successfully treated abdominal pregnancy causing hemoperitoneum using combined surgical and systemic methotrexate therapy: a case report and review of cases treated at Kyushu University Hospital. Gynecol Obstet Invest 2013; 76: 188192. doi: $10.1159 / 000353980$
[61] Brouard K], Howard BR, Dyer RA. Hepatic Pregnancy Suspected at Term and Successful Delivery of a Live Neonate With Placental Attachment to the Right Lobe of the Liver. Obstet Gynecol 2015; 126: 207-210. doi:10.1097/aog.0000000000000703

[62] Chin PS, Wee HY, Chern BS. Laparoscopic management of primary hepatic pregnancy. Aust N Z J Obstet Gynaecol 2010; 50: 95-98. doi:10.1111/j.1479-828X.2009.01113.x

[63] Guo B, Li D. Hepatobiliary and Pancreatic: Ectopic pregnancy in the liver: another rare cause of space occupying lesion. J Gastroenterol Hepatol 2016; 31: 1514. doi:10.1111/jgh.13396

[64] Hao J, Cheng Z, Hu N et al. Imaging of Hepatic Ectopic Pregnancy by 18F-FDG PET/CT. Clin Nucl Med 2016; 41: 697-698. doi:10.1097| rlu. 0000000000001307

[65] Hu S, Song Q, Chen K et al. Contrast-enhanced multiphasic CT and MR of primary hepatic pregnancy: a case report and literature review. Abdom Imaging 2014; 39: 731-735. doi:10.1007/s00261-014-0101-5

[66] Kuai XP, Wang SY, Qiu JM. Ectopic pregnancy implanted in the liver under the diaphragm. Taiwan J Obstet Gynecol 2013; 52: 586-587. doi:10.1016/j.tjog.2013.10.024

[67] Ma J, Zhou C, Duan Z et al. Successful management of primary hepatic pregnancy with selective hepatic artery embolization and intra-arterial methotrexate infusion. Int | Gynaecol Obstet 2013; 122: 78-79. doi:10.1016/j.ijgo.2013.02.019

[68] Moores KL, Keriakos RH, Anumba DO et al. Management challenges of a live 12-week sub-hepatic intra-abdominal pregnancy. BJOG 2010; 117: 365-368. doi:10.1111/j.1471-0528.2009.02450.x

[69] Qiao JC, Chang ZG, Wei JM et al. Hepatic ectopic pregnancy treated successfully by hepatectomy. Chin Med J 2013; 126: 4806-4807

[70] Ramphal SR, Moodley J, Rajaruthnam D. Hepatic pregnancy managed conservatively. Trop Doct 2010; 40: 121-122. doi:10.1258/td.2009. 090218

[71] Sibetcheu Tchatou A, Tchounzou R, Mbuagbaw L et al. Successful medical treatment of a hepatic pregnancy: a case report. J Med Case Rep 2017; 11: 70. doi:10.1186/s13256-017-1227-1

[72] Wang C, Cheng L, Zhang Z et al. Imaging diagnosis of hepatic ectopic pregnancy: A report of one case. Intractable Rare Dis Res 2012; 1: 40 44. doi:10.5582/irdr.2012.v1.1.40

[73] Yadav R, Raghunandan C, Agarwal S et al. Primary hepatic pregnancy. J Emerg Trauma Shock 2012; 5: 367-369. doi:10.4103/09742700.102417

[74] Zhao RF, Huang SR, Xu LL et al. Successful Management of a Live 14week Primary Hepatic Ectopic Pregnancy Combined with a Residual Horn of the Uterus Using Laparoscopy. Chin Med J 2017; 130: 30133014. doi:10.4103/0366-6999.220298

[75] Allen WL, Subba B, Yoong W et al. Chronic abdominal pregnancy following rupture from a bicornuate uterus. Arch Gynecol Obstet 2007; 275: 393-395. doi:10.1007/s00404-006-0249-5

[76] Bajis R, Paterson D, McElhinney B. Primary omental ectopic pregnancy: a case report. J Obstet Gynaecol 2019; 39: 560-561

[77] Behjati S, Bamfo JE, Bates AW et al. A near miss: lessons from a case of omental pregnancy. J Obstet Gynaecol 2009; 29: 559-560. doi:10.1080 01443610902984946

[78] Chen L, Qiu L, Diao X et al. CT findings of omental pregnancy: a case report. Jpn J Radiol 2015; 33: 499-502. doi:10.1007/s11604-015-0449-7

[79] Chopra S, Keepanasseril A, Suri V et al. Primary omental pregnancy: case report and review of literature. Arch Gynecol Obstet 2009; 279: 441442. doi:10.1007/s00404-008-0730-4

[80] da Silva BB, de Araujo EP, Cronemberger JN et al. Primary twin omental pregnancy: report of a rare case and literature review. Fertil Steril 2008; 90: 2006.e13-2006.e15. doi:10.1016/j.fertnstert.2008.03.038 
[81] Maiorana A, Incandela D, Giambanco L et al. Omental pregnancy: case report and review of literature. Pan Afr Med J 2014; 19: 244. doi:10.11604/pamj.2014.19.244.3661

[82] Seol HJ, Kim T, Lee SK. Successful laparoscopic management of primary omental pregnancy. Arch Gynecol Obstet 2010; 281: 163-165. doi:10.1007/s00404-009-1100-6

[83] Srinivasan A, Millican S. Laparoscopic management of an abdominal pregnancy. Case Rep Obstet Gynecol 2014; 2014: 562731. doi: $10.1155 / 2014 / 562731$

[84] Takeda A, Nakamura H, Hayashi S et al. Primary Omental Pregnancy: Successful Laparoendoscopic Single-site Partial Omentectomy After Diagnosis by Diffusion-weighted Magnetic Resonance Imaging. J Minim Invasive Gynecol 2016; 23: 6-7. doi:10.1016/j.jmig.2015.04.009

[85] Tanase $\mathrm{Y}$, Yoshida S, Furukawa $\mathrm{N}$ et al. Successful laparoscopic management of a primary omental pregnancy: case report and review of literature. Asian J Endosc Surg 2013; 6: 327-329. doi:10.1111/ases.12056

[86] Yip SL, Tan WK, Tan LK. Primary omental pregnancy. BM] Case Rep 2016; 2016: bcr2016217327. doi:10.1136/bcr-2016-217327

[87] Demendi C, Langmar Z, Banhidy F et al. Successful operative management of an intact second trimester abdominal pregnancy with additional preoperative selective catheter embolization and postoperative methotrexate therapy. Med Sci Monit 2011; 17: Cs53-Cs55. doi:10.12659/ msm. 881752

[88] Pichaichanlert A, Luvira V, Tipsunthonsak N. Abdominal Pregnancy in the Small Intestine Presenting as Acute Massive Lower Gastrointestinal Hemorrhage. Case Rep Surg 2017; 2017: 8017937. doi:10.1155/2017| 8017937

[89] Salathiel DT, Anicet Z, Madoue GB et al. Abdominal pregnancy discovered during laparotomy for complications after attempted abortion. South Sudan Medical Journal 2016; 16: 14

[90] Thompson R], Hawe M]. A rare pathological trinity: an appendiceal ectopic pregnancy, acute appendicitis and a carcinoid tumour. Ir ] Med Sci 2011; 180: 579-580. doi:10.1007/s11845-009-0283-y

[91] Trail CE, Watson A, Schofield AM. Case of hepatic flexure ectopic pregnancy medically managed with methotrexate. BM] Case Rep 2018; 2018: bcr2017220480. doi:10.1136/bcr-2017-220480

[92] Yildizhan R, Kolusari A, Adali F et al. Primary abdominal ectopic pregnancy: a case report. Cases J 2009; 2: 8485. doi:10.4076/1757-1626-28485

[93] Biolchini F, Giunta A, Bigi L et al. Emergency laparoscopic splenectomy for haemoperitoneum because of ruptured primary splenic pregnancy: a case report and review of literature. ANZ J Surg 2010; 80: 55-57. doi:10.1111/j.1445-2197.2009.05176.x

[94] Gao H, Yuan T, Ding Y et al. Primary splenic pregnancy with hemorrhagic shock. J Obstet Gynaecol Res 2017; 43: 1342-1345. doi:10.1111/ jog. 13348

[95] Greenbaum A, Miskimins R, Coffman B et al. Management of splenic ectopic pregnancy presenting with massive haemoperitoneum. BMJ Case Rep 2016; 2016: bcr2016218291. doi:10.1136/bcr-2016-218291

[96] Klang E, Keddel N, Inbar Y et al. Splenic Pregnancy: A New Minimally Invasive Approach to Treatment. Cardiovasc Intervent Radiol 2016; 39: 1339-1342. doi:10.1007/s00270-016-1403-5

[97] Perez A, Caban-Martinez AJ, Alvarez W et al. Subsplenic ectopic pregnancy adjacent to a rudimentary uterine horn. Int J Gynaecol Obstet 2008; 102: 78-79. doi:10.1016/j.ijgo.2008.02.014

[98] Python JL, Wakefield BW, Kondo KL et al. Ultrasound-Guided Percutaneous Management of Splenic Ectopic Pregnancy. J Minim Invasive Gynecol 2016; 23: 997-1002. doi:10.1016/j.jmig.2016.05.004
[99] Rathore R, Shilpi S, Chopra R et al. Primary Splenic Pregnancy- A Rare but Imperative Cause of Hemoperitoneum - Case Report and Review of Literature. Turk Patoloji Derg 2019; 35: 242-246. doi:10.5146/ tjpath.2017.01403

[100] Wu BQ, Zhu F, Jiang Y et al. Case of spontaneous splenic rupture caused by ectopic pregnancy in the spleen. J Obstet Gynaecol Res 2017; 43: 1778-1780. doi:10.1111/jog. 13440

[101] Wu L, Jiang X, Ni J. Successful diagnosis and treatment of early splenic ectopic pregnancy: A case report. Medicine 2018; 97: e0466. doi:10.1097/md.0000000000010466

[102] Anderson PM, Opfer EK, Busch JM et al. An Early Abdominal Wall Ectopic Pregnancy Successfully Treated with Ultrasound Guided Intralesional Methotrexate: A Case Report. Obstet Gynecol Int 2009; 2009: 3. doi: $10.1155 / 2009 / 247452$

[103] Borton Z, Landon AG, Singh S. A painless pelvic wall ectopic pregnancy. BMJ Case Rep 2015; 2015: bcr2015211529. doi:10.1136/bcr-2015211529

[104] Gorry A, Morelli ML, Olowu O et al. Laparoscopic management of abdominal ectopic pregnancy using FLOSEAL Hemostatic Matrix. Int J Gynaecol Obstet 2012; 117: 83-84. doi:10.1016/j.ijgo.2011.11.003

[105] Irani M, Elias RT, Pereira N et al. Abdominal ectopic pregnancy with undetectable serum beta-human chorionic gonadotropin 9 days following blastocyst transfer. J Obstet Gynaecol Res 2016; 42: 1886-1888. doi: $10.1111 /$ jog. 13127

[106] Lee C. Abdominal pregnancy in a low-resource setting. Obstet Gynecol 2015; 125: 1039-1041. doi:10.1097/aog.0000000000000706

[107] Chen L, Liu J, Shu J et al. Successful laparoscopic management of diaphragmatic pregnancy:a rare case report and brief review of literature. BMC Pregnancy Childbirth 2019; 19: 99. doi:10.1186/s12884019-2248-0

[108] Noguchi D, Matsumoto N, Kamata S et al. Ectopic pregnancy developing in a cyst of the canal of Nuck. Obstet Gynecol 2014; 123: 472-476. doi:10.1097/aog.0000000000000099

[109] Iwama H, Tsutsumi S, Igarashi H et al. A case of retroperitoneal ectopic pregnancy following IVF-ET in a patient with previous bilateral salpingectomy. Am J Perinatol 2008; 25: 33-36. doi:10.1055/s-20071004829

[110] Jiang W, Lv S, Sun L et al. Diagnosis and treatment of retroperitoneal ectopic pregnancy: review of the literature. Gynecol Obstet Invest 2014; 77: 205-210. doi:10.1159/000353691

[111] Ouassour S, Filali AA, Raiss M et al. Retroperitoneal Ectopic Pregnancy: Diagnosis and Therapeutic Challenges. Case Rep Surg 2017; 2017: 9871865. doi:10.1155/2017/9871865

[112] Pak JO, Durfee JK, Pedro L et al. Retroperitoneal Ectopic Pregnancy. Obstet Gynecol 2018; 132: 1491-1493. doi:10.1097/aog.000000000 0002965

[113] Yang $Y$, Liu Z, Song $L$ et al. Diagnosis and surgical therapy of the retroperitoneal ectopic pregnancy: A case report. Int J Surg Case Rep 2018; 49: 21-24. doi:10.1016/j.ijscr.2018.05.027

[114] Zhang M, Qin LL. A case of retroperitoneal para-aortic ectopic pregnancy detected by sonography. J Clin Ultrasound 2018; 46: 412-414. doi:10.1002/jcu.22554

[115] Martínez-Varea A, Hidalgo-Mora JJ, Payá V et al. Retroperitoneal ectopic pregnancy after intrauterine insemination. Fertil Steril 2011; 95: 2433.e1-2433.e3. doi:10.1016/j.fertnstert.2011.02.026

[116] Yang M, Cidan L, Zhang D. Retroperitoneal ectopic pregnancy: a case report and review of the literature. BMC Pregnancy Childbirth 2017; 17: 358. doi:10.1186/s12884-017-1542-y

[117] Lin JX, Liu Q, Ju Y et al. Primary obturator foramen pregnancy: a case report and review of literature. Chin Med J 2008; 121: 1328-1330 
[118] Chishima F, Kato-Suzuki E, Ichikawa G et al. Rare case of primary peritoneal pregnancy infiltrated into the Gerota's fascia of the right kidney. J Obstet Gynaecol Res 2013; 39: 1073-1076. doi:10.1111/ j.1447-0756.2012.02057.x

[119] Guan Z, Li HF, Guo LL et al. Management of pancreatic ectopic pregnancy. Taiwan J Obstet Gynecol 2015; 54: 629-631. doi:10.1016/ j.tjog.2015.08.022
[120] Meire I, van Heusden A, Roukema MS et al. A retroperitoneal pregnancy of an anencephalic fetus. J Obstet Gynaecol 2007; 27: 518519. doi:10.1080/01443610701465071

[121] Lockhat F, Corr P, Ramphal S et al. The value of magnetic resonance imaging in the diagnosis and management of extra-uterine abdominal pregnancy. Clin Radiol 2006; 61: 264-269. doi:10.1016/j.crad.2005. 10.013 